\title{
Morphologies of multicompartment micelles formed by triblock copolymers
}

\author{
Shih-Hao Chou \\ Department of Chemical Engineering, National Taiwan University, Taipei, Taiwan 106, Republic of China \\ Heng-Kwong Tsao ${ }^{\text {a),b) }}$ \\ Department of Chemical and Materials Engineering, Institute of Materials Science and Engineering, \\ National Central University, Jhongli, Taiwan 320, Republic of China \\ Yu-Jane Sheng ${ }^{\mathrm{a}), \mathrm{c})}$ \\ Department of Chemical Engineering, National Taiwan University, Taipei, Taiwan 106, Republic of China
}

(Received 22 August 2006; accepted 16 October 2006; published online 16 November 2006)

\begin{abstract}
Multicompartment micelles are desirable for advanced applications such as drug delivery. Recently, core-shell-corona (CSC) and segmented-worm (SW) micelles formed by $A B C$ triblock terpolymers with three mutually immiscible blocks are observed in experiments. We have performed dissipative particle dynamics simulations to study the effects of molecular architecture, block length, and solution concentration on the morphologies of $A B C$ triblock terpolymers. The formation of CSC and SW micelles for linear and miktoarm star $A B C$ terpolymers is confirmed in this work. In addition, we predict that different multicompartment micellar morphologies (e.g., incomplete skin-layered micelles and segmented worms) can be formed by linear copolymer with different arrangements of the three blocks. (C) 2006 American Institute of Physics. [DOI: 10.1063/1.2390716]
\end{abstract}

\section{INTRODUCTION}

The self-assembly of block copolymers in a selective solvent is of fundamental interest ${ }^{1}$ and offers tremendous promise for advanced applications, such as nanomaterial synthesis, potential delivery vehicles for pharmaceuticals, and gene therapy agent. Despite the particular morphology (e.g., spheres, cylinders, and vesicles), those aggregates generally consist of two compartments: the micellar core and the micellar corona. However, the formation of multicompartment micelles is desirable. ${ }^{2}$ The complex systems consisting of several compartments of different characters are ubiquitous in the living nature. For example, a single cell containing many different units to perform distinct biological functions is the quintessential example. In a multicompartment micelle, two or more active but incompatible agents could be stored in distinct nanodomains and then delivered to the same place at the same time for drug delivery applications. ${ }^{3}$

It is appealing to study whether rather simple multiblock copolymer architectures can be designed to create structures within structures. ${ }^{3-6}$ Since aggregates formed by amphiphilic $A B$ diblock or $A B A$ triblock copolymers provide only two compartments, $A B C$ triblock polymer with three mutually immiscible blocks can serve as a minimal model system to form several compartments in one micelle. For example, $A$ is hydrophobic block based on fluorocarbons, $B$ is a hydrophobic block based on hydrocarbons, and $C$ is a hydrophilic block. The structured micelles are possibly formed as assemblies in which separate $A$ and $B$ core domains exist within a solvated $C$ corona. $^{7}$ Recently, some progresses toward this goal have been reported for linear and star terpolymers. The

\footnotetext{
a) Authors to whom correspondence should be addressed.

${ }^{b)}$ Electronic mail: hktsao@cc.ncu.edu.tw

${ }^{c)}$ Electronic mail: yjsheng@ntu.edu.tw
}

formation of "three-layer" micelles in water by linear $A B C$ triblocks has been observed. These micelles, referred to as core-shell-corona (CSC) micelles, include spheres and disks. ${ }^{4,8}$ The subdivision of the micellar interior contains two concentric nanodomains. On the other hand, depending on the relative block lengths, miktoarm star $A B C$ terpolymer can form discrete, multidomain cores or extended, segmented-worm (SW) structures. ${ }^{3,5}$ More recently, the miktoarm star terpolymers were found to be able to selfassemble into forming vesicles with laterally nanostructured membrane. ${ }^{9}$ The lateral structure consists of approximately hexagonally packed fluorocarbon channels immersed in a continuous, two-dimensional hydrocarbon bilayer.

The experimental results demonstrate that there is a large parameter space (e.g., block lengths, choice of monomers, and chain architecture) to explore the formation of multicompartment micelles with different morphologies. Molecular simulation is able to provide more microscopic level information than experiment. Although micellization of block copolymers has been studied since the 1960s, little simulation work has been done so far on the topics of micelles formed by copolymers. Also, little is known about their exact internal structures. Classical molecular dynamics (MD) and Monte Carlo simulations can only be applied to study systems at limited length and time scales. However, larger scale simulation is critical for the investigation of polymeric behaviors. The dissipative particle dynamics (DPD) is a mesoscopic simulation technique which can treat a wider range of length and time scales compared to atomistic simulations. It has been successfully used to study morphologies and physical properties of polymer as well as surfactant solutions. For example, in a recent work of DPD simulations,${ }^{10}$ the method has been applied to investigate the formation and the rupture mechanism of vesicles (polymersomes) formed by the block 
copolymer. Morphological studies of multicompartment micelles are of great importance to complement experimental observations and advanced biomedical applications. In this work we perform DPD to explore the morphologies of multicompartment micelles formed by $A B C$ terpolymers in aqueous solutions. The effects of molecular architecture, block length, and polymer concentration on the morphologies are examined.

\section{MODEL AND SIMULATION METHODS}

The DPD method, introduced by Hoogerbrugge and Koelman in $1992,{ }^{11}$ is a particle-based, mesoscale simulation technique. This method combines some of the detailed description of the MD but allows the simulation of hydrodynamic behavior in much larger, complex systems, up to the microsecond range. Like MD, the DPD particles obey Newton's equation of motion,

$$
\frac{d \mathbf{r}_{i}}{d t}=\mathbf{v}_{i}, \quad \frac{d \mathbf{v}_{i}}{d t}=\mathbf{f}_{i},
$$

where $\mathbf{f}_{i}$ denotes the total forces acting on particle $i$. In the system, there are four different species of DPD particles, including solvent $(S)$, highly hydrophobic particle $(A)$, hydrophobic particle $(B)$, and hydrophilic particle $(C)$.

The force is composed of three different pairwiseadditive forces: conservative $\left(\mathbf{F}^{C}\right)$, dissipative $\left(\mathbf{F}^{D}\right)$, and random forces $\left(\mathbf{F}^{R}\right)$,

$$
\mathbf{f}_{i}=\sum_{j \neq i}\left(\mathbf{F}_{i j}^{C}+\mathbf{F}_{i j}^{D}+\mathbf{F}_{i j}^{R}\right)
$$

These forces conserve net momentum and all acts along the line joining the two particles. The conservative force $\mathbf{F}^{C}$ for nonbonded beads is a soft-repulsive force,

$$
\mathbf{F}_{i j}^{C}=\left\{\begin{array}{cc}
a_{i j}\left(1-r_{i j}\right) \hat{\mathbf{r}}_{i j}, & r_{i j}<1 \\
0, & r_{i j}>1,
\end{array}\right.
$$

where $a_{i j}$ is a maximum repulsion between particles $i$ and $j$ and $r_{i j}$ is the magnitude of the bead-bead vector. $\hat{\mathbf{r}}_{i j}$ is the unit vector joining beads $i$ and $j$. The dissipative or drag force has the form

$$
\mathbf{F}_{i j}^{D}=-\gamma w^{D}\left(r_{i j}\right)\left(\hat{\mathbf{r}}_{i j} \cdot \mathbf{v}_{i j}\right) \hat{\mathbf{r}}_{i j},
$$

where $\mathbf{v}_{i j}=\mathbf{v}_{i}-\mathbf{v}_{j}$ and $w^{D}$ is a $r$-dependent weight function. The form is chosen to conserve the total momentum of each pair of particles, and therefore the total momentum of the system is conserved. The dissipative force acts to reduce the relative momentum between particles $i$ and $j$, while random force is to impel energy into the system. The random force also acts between all pairs of particles as

$$
\mathbf{F}_{i j}^{R}=\sigma w^{R}\left(r_{i j}\right) \theta_{i j} \hat{\mathbf{r}}_{i j},
$$

where $w^{R}$ is also a $r$-dependent weight function and $\theta_{i j}$ is a randomly fluctuating variable with Gaussian statistics,

$$
\left\langle\theta_{i j}(t)\right\rangle=0, \quad\left\langle\theta_{i j}(t) \theta_{k l}\left(t^{\prime}\right)\right\rangle=\left(\delta_{i k} \delta_{j l}+\delta_{i l} \delta_{j k}\right) \delta\left(t-t^{\prime}\right) .
$$

Note that in Brownian dynamics white noise is added to the equation of motion for each particle independently. However, in DPD simulation, the random force is acting along the line of center of each pair of beads, and therefore conserves the total momentum even it puts in energy to the system. Español and Warren ${ }^{12}$ have shown that in order to satisfy the fluctuation-dissipation theorem and for the system to evolve to a steady state that corresponds to the Gibbs canonical ensemble, only one of the two weight functions can be chosen arbitrarily and this choice fixes the other weight function. There is also a relation between the amplitudes $(\sigma$ and $\gamma)$ and $k_{B} T$. Therefore,

$$
\begin{aligned}
& w^{D}(r)=\left[w^{R}(r)\right]^{2}, \\
& \sigma^{2}=2 \gamma k_{B} T .
\end{aligned}
$$

According to Groot and Warren, ${ }^{13}$ we choose

$$
w^{D}(r)=\left[w^{R}(r)\right]^{2}=\left\{\begin{array}{cc}
(1-r)^{2}, & r<1 \\
0, & r \geqslant 1 .
\end{array}\right.
$$

For polymer systems, we also have to consider the interaction forces between bonded beads,

$$
\mathbf{F}_{i, \text { spring }}^{C}=-\sum_{j} C\left(r_{i j}-r_{\mathrm{eq}}\right) \hat{\mathbf{r}}_{i j}
$$

where the sum runs over all particles to which particle $i$ is connected. In this work, we have chosen $C=4$ and $r_{\text {eq }}=0.0$. This choice is only a coarse-graining selection to show the constraint imposed upon connected beads of a polymeric chain. This choice of $C$ and $r_{\text {eq }}$ will not affect the qualitative behavior of the systems studied in our work.

In DPD simulations, the masses of the beads are set as 1 , therefore the force acting on a bead equals its acceleration. Also, as we can see, all the forces except for the bonded spring force come to zero outside a certain cutoff radius $r_{c}$, where $r_{c}=1$ in this work.

In this work, we consider an aqueous solution $(W)$ of triblock copolymer representing approximately poly(ethylene oxide) $(O)$, polyethylethylene $(E)$, and poly(perfluoropropylene oxide) $(F)$. The triblock copolymer is designated $A B C(a-b-c)$, where $a, b$, and $c$ denote the number of DPD particles associated with $A, B$, and $C$ blocks, respectively. To compare with the experimental observation, we investigate miktoarm star $O E F(x-6-7)$ and linear $O E F(x-6-7)$, where $x$ varies from 10 to 20 .

The interaction parameters chosen are $a_{O E}=40, a_{O F}$ $=45, a_{E F}=42.5, a_{O W}=25.3, a_{E W}=50$, and $a_{F W}=60$. Note that these interaction parameters $\left(a_{i j}\right)$ are not randomly selected. They are so chosen in an attempt to retain the characteristic interactions associated with water $(W)$, poly(ethylene oxide) $(O)$, polyethylethylene $(E)$, and poly(perfluoropropylene oxide) $(F)$. These interaction parameters can be estimated based on the relationship between $a$ and the Flory-Huggins parameter $\chi$ established by Groot and Warren, ${ }^{13}$ for density $\rho=3$,

$$
a_{i j}=a_{i i}+3.497 \chi_{i j}
$$

where $a_{i i}=25$ leads to the compressibility of water. Therefore, if species $i$ and $j$ are fairly compatible, $\chi_{i j} \approx 0$ and $a_{i j}$ $\approx 25$. As incompatibility between $i$ and $j$ increases, $a_{i j}$ increases. The $\chi$ parameters for different pair species can be 
(a)

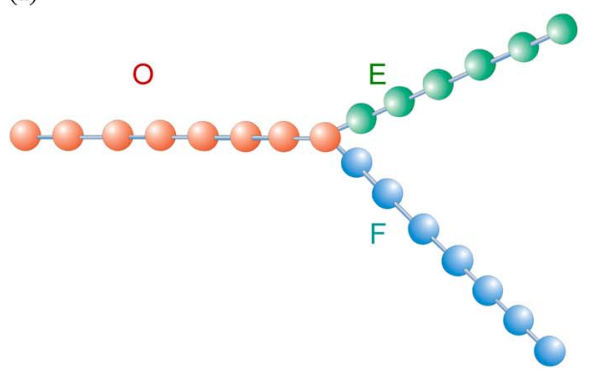

(b)

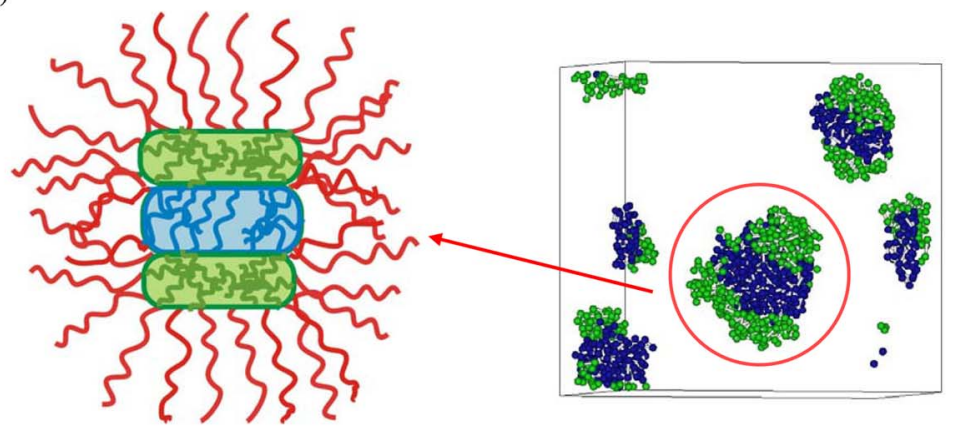

FIG. 1. (Color online) (a) Schematic representation of a miktoarm star $O E F$. (b) Schematic representation and simulation morphologies of hamburger micelles formed by $O E F$ (20-6-7) stars. (c) Segmented-worm and (d) network formation by segmented worms. $O$ (red), $E$ (green), and $F$ (blue). For clarity, we remove $O$ blocks in the snapshots.

(c)

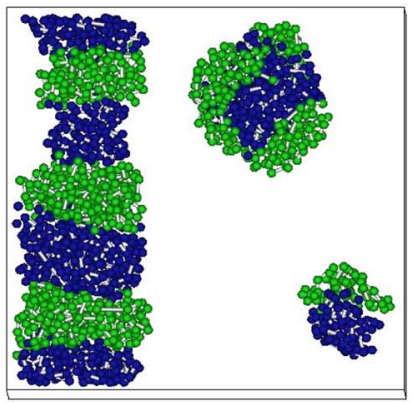

(d)

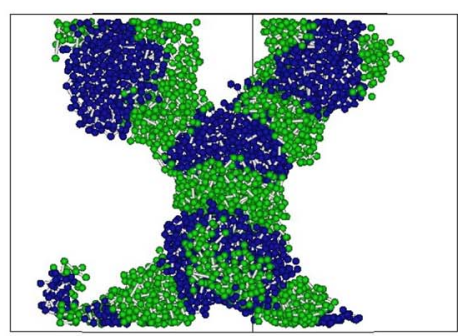

estimated from many sources such as commercially available software "MATERIALS STUDIO." 14 The $\chi$ parameter is provided by performing simulations of molecular fragments retaining all atomistic details. For example, $\chi_{O E}$ value is estimated to be about 1.01, indicating that it is rather incompatible between monomers of $O$ and $E$. By grouping approximately three to five monomers into a DPD bead, $\chi_{O E}$ increases four times to be about 4.04. As a result, from Eq. (10), $a_{O E} \approx 40$. The incompatibility between $O$ and $F$ is slightly greater than that of $O$ and $E$; therefore $a_{O F}=45$ is selected. Also, since $O$ is rather hydrophilic, we have chosen $a_{O W}=25.3$. Polyethylethylene $(E)$, on the other hand, is known to be quite hydrophobic as $\chi_{W E}$ is estimated to be about 2.86. The grouping effects increase the $\chi$ value and thus $a_{W E}=50$ is used. Also, the $F$ block is more hydrophobic than the $E$ block, and as a consequence, $a_{F W}=60$ seem to be a reasonable choice. These parameters reveal the mutual incompatibility among the $O E F$ three blocks and point out the fact that $O$ is a hydrophilic block and $F$ block is more hydrophobic than $E$ block.

On the basis of the algorithm described, the dynamics of 24000 DPD particles, starting from random distribution, was simulated in a cubic box $\left(20^{3}\right.$ or $\left.30^{3}\right)$ under periodic boundary conditions. The equation of motion are integrated with a modified velocity Verlet algorithm $^{13}$ with $\lambda=0.65$ and $\Delta t$ $=0.05$. In this work, each simulation takes at least 200000 steps and the first 80000 steps are for equilibration. Note that DPD simulation utilizes soft-repulsive potentials, and the systems studied are allowed to evolve much faster than the "brute-force" molecular dynamics. Therefore a typical DPD simulation needs only about 50 000-100 000 steps to equilibrate. After that the resulting morphologies are analyzed. The simulation is performed by using a serial FORTRAN code on a personal computer (PC) with CPU of Pentium d-930. It takes about $72 \mathrm{~h}$ of CPU time for a given simulation run with box size equal to 30 .

\section{RESULTS AND DISCUSSION}

Multicompartment micelles have been experimentally observed in dilute triblock copolymer solutions. Based on the fitting of the scattering analyses, such as small-angle X-ray and neutron scatterings, and observation with transmission electron microscopy (TEM), ${ }^{3,8}$ structured micelles such as core/shell/corona and segmented-worm morphologies are inferred. Molecular simulation is an alternative approach to gain more direct and microscopic level information than experiments. The building of mesoscale micellar solutions with 
the use of classical molecular dynamics at atomic resolution is a challenge at present, owing to the length and time scales at which these phenomena can occur. However, mesoscale simulations such as dissipative particle dynamics can treat a wide range of length and time scales by many orders of magnitude compared to atomistic simulations. In this paper, DPD is performed to explore the morphologies of structured micelles formed by $A B C$ triblock terpolymer with three mutually immiscible blocks, including miktoarm star and linear architectures.

It was experimentally reported that miktoarm star terpolymers $O E F$, as schematically illustrated in Fig. 1(a), can form "hamburger" micelles and "segmented-worm" micelles by varying the length of hydrophilic block $(O)$. Those structured micelles are also observed by DPD recently for star copolymer of relatively short block lengths $O E F(x-4-2)$ with the volume fraction at $0.1{ }^{15}$ In their study, the repulsive interactions between $F$ block and others are very strong, i.e., $a_{W F}=120, a_{E F}=75$, and $a_{O F}=90$. In the current study, we examine the structure of the micelle formed by star copolymer $O E F(x-6-7)$ of comparatively long block lengths but with weaker repulsions for $a_{X F}$, where $X=O, E$, or $W$. This choice allows the formation of micelles with large enough hydrophobic domains that can be clearly identified. We focus on the effect of the copolymer concentration on the micellar structures in addition to varying the length of the $O$ block.

For the miktoarm star with a fixed length of $O$ block, i.e., $O E F$ (20-6-7), both discrete hamburger micelles [Fig. 1(b)] and segmented-worm micelles [Fig. 1(c)] can be observed depending on the polymer concentration. The discrete micelle is formed at very low concentration and consists of a $F$ core, surrounded by a few $E$ nanodomains. In general, two $E$ nanodomains are found on top and bottom of the $F$ core to form $E F E$ hamburger micelles [Fig. 1(b)]. The $O$ blocks emanate from the $E-F$ interface and curl around to protect the hydrophobic core. As polymeric concentration increases, elongated, wormlike structures emerge [Fig. 1(c)]. The worms are layered with alternating sections of $F$ and $E$ blocks along the long axis. The $O$ coronas are shared by $E$ and $F$ layers to shield them from the highly unfavorable exposure to water. The segmented-worm micelle is always formed for star ( $x-6-7)$ as long as the polymer concentration is high enough. The onset concentration for the formation of segmented-worm micelles is increased with the hydrophilic $O$ block length. When the hydrophilic $O$ block is large $(x=20)$, the $O$ block screen the hydrophobic core effectively and the hamburger micelles can survive at higher concentration. On the other hand, for stars with shorter $O$ blocks $(x=10)$, segmented worms form at a much lower concentration. This is because by forming into segmented worms can the hydrophobic blocks ( $E$ and $F$ ) be able to share their $O$ coronas and avoid direct contact with the solvents. When the concentration is even higher, the segmented micelles may join together to form segmented network, as illustrated in Fig. 1(d).

Our simulation results indicate that $O E F$ star copolymer with a given composition (i.e., the length of each individual block is fixed) may form different micellar morphologies by varying the polymer concentration. Altering the copolymer (a)

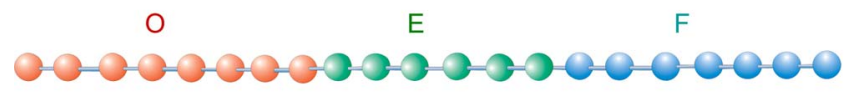

(b)

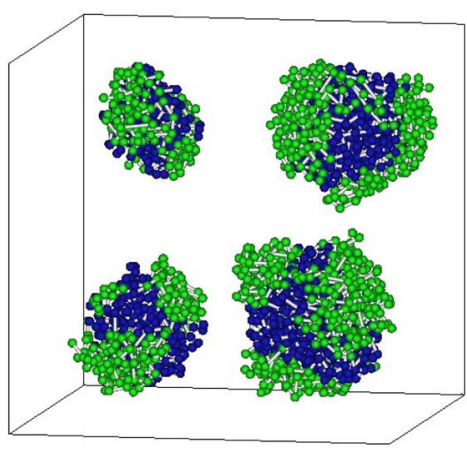

(c)
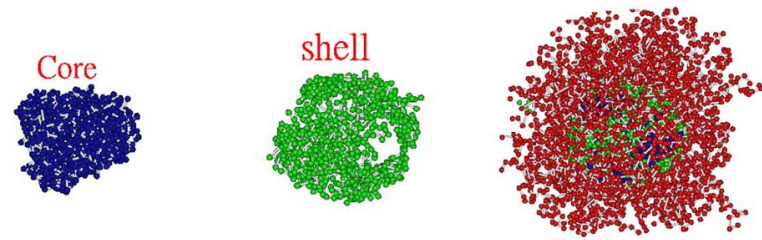

FIG. 2. (Color online) (a) Schematic representation of a linear triblock copolymer $O E F$. (b) Simulation results of micelles formed by linear $O E F$ (20-6-7). For clarity, we remove $O$ blocks in the snapshots. (c) Morphology of a single core/shell/corona micelle formed by linear $O E F$ (20-6-7).

composition just influences the onset concentration associated with a particular morphology. It should be emphasized that the change of the micellar morphology by varying polymer concentration is not like the first-order transition at all. We often observe micelles with different morphologies coexisting at a given polymer concentration. For example, both hamburger and segmented-worm micelles are observed in Fig. 1(c). However, the ratio of the two different micellar species will gradually change with the concentration. This simulation result is consistent with experimental observation. $^{3,9}$ As shown in cryo-TEM images for miktoarm star, different micellar morphologies (hamburger and segmented worm) can coexist. If one regards a hamburger micelle as the elementary unit of a segmented-worm micelle, then only one type of micelle exists in the solution but with a very wide size distribution (large polydispersity). Nevertheless, the size distribution will vary with polymer concentration. Since the crossover between different micellar morphologies is not a discontinuous phase transition, a conventional phase diagram cannot be defined simply. In general, Figs. 1(b)-1(d) illustrate the morphology of miktoarm star changes mainly from hamburger, segmentedworm micelle, to segmented-worm network as the copolymer concentration is increased.

In addition to miktoarm star, various morphologies of multicompartment micelles can be disclosed by linear triblock copolymers with different arrangements of the three blocks, $O E F, O F E$, and $E O F$. The schematic representations 
(a)

O

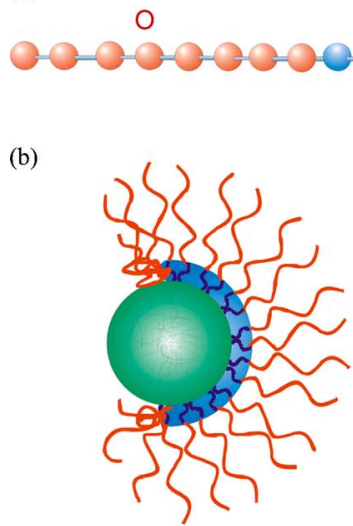

$\mathrm{F}$

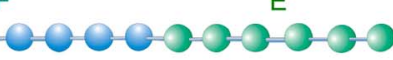

(c)

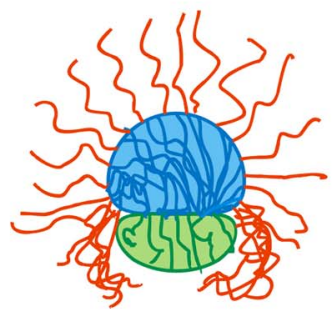

(d)
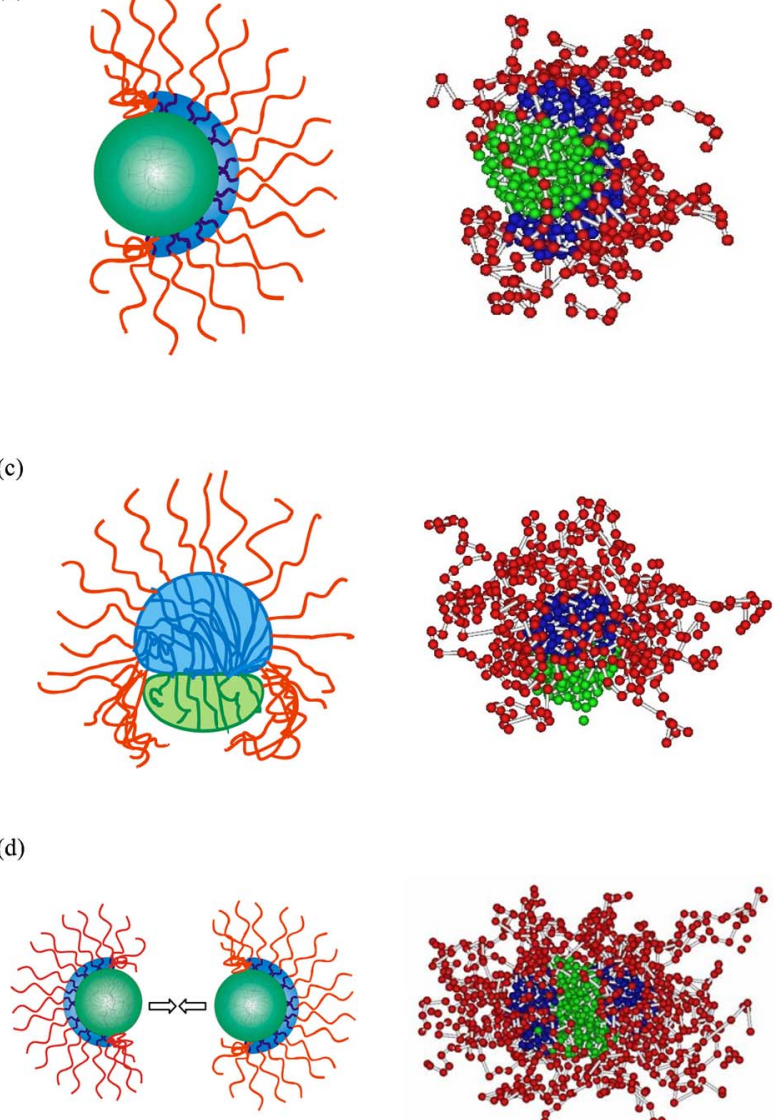

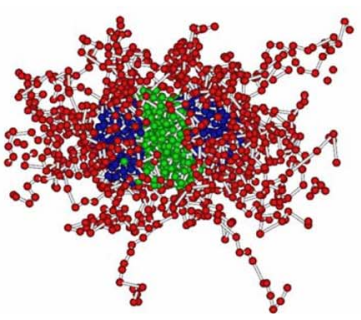

FIG. 3. (Color online) (a) Schematic representation of a linear triblock copolymer OFE. (b) Schematic representation and simulation morphology of the incomplete skin-layered micelles formed by linear OFE (10-7-6) and (c) schematic representation and simulation morphology of the bicore micelles formed by linear OFE (20-7-6). (d) Schematic representation and simulation morphology of the hamburger micelles formed by linear $O F E$ (10-7-6) at high concentration.

of linear $O E F, O F E$, and $E O F$ are shown in Figs. 2(a), 3(a), and 4(a), respectively. For linear $O E F$ ( $x$-6-7) triblocks, the core-shell-corona structure can be evidently identified with the $F$ core, $E$ shell, and $O$ corona, as shown in Figs. 2(b) and 2(c). In the experimental works, ${ }^{4,8}$ the morphology of sphere or ellipsoid has been observed by TEM or atomic force microscopy for linear triblock copolymer, e.g., poly(ethylene oxide)- $b$-poly(styrene)- $b$-1,2-poly(butadiene): $\mathrm{C}_{6} \mathrm{~F}_{13} \mathrm{I}$. Owing to the mutual immiscibility among the three blocks, it is inferred that the multilayer CSC micelles are formed. In our simulations, we directly observe this CSC morphology, which is consistent with the experimental findings. Nonetheless, the spread of the $E$ blocks on the surface of the $F$ core is not uniform. This result is mainly due to the mutual incompatibility between $E$ and $F$. The competition between energetic and entropic effects leads to a partial spread of the $E$ blocks around $F$ cores. This outcome is, in fact, energetically favorable as long as $a_{O E}$ is comparable with $a_{O F}$ and (a)

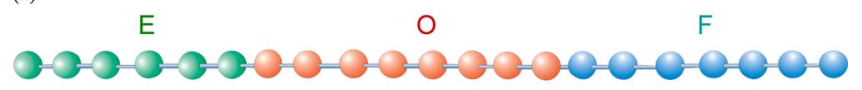

(b)

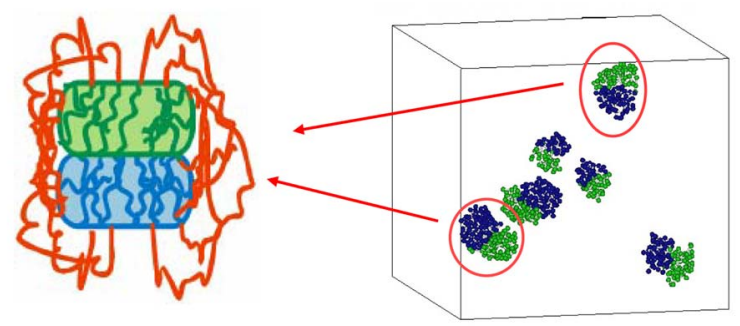

(c)
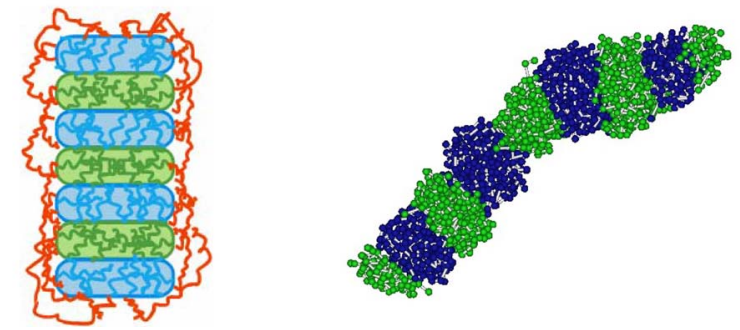

FIG. 4. (Color online) (a) Schematic representation of a linear triblock copolymer EOF. (b) Schematic representation and simulation morphologies of micelles formed by (a) linear EOF (6-20-7) and (c) linear EOF (6-10-7). For clarity, we remove $O$ blocks in the snapshots.

the $O$ blocks can serve as a shielding layer preventing the $E$ and $F$ blocks from interacting with water. In general, the aggregation number of the CSC micelle declines as the length of $O$ block $(x-6-7)$ is increased.

It is interesting to find that rather different morphologies can be observed by changing the arrangement of the $O, E$, and $F$ blocks even for the same composition in a triblock copolymer. For linear $O F E$ ( $x-7-6)$ with shorter $O$ blocks, the $E$ blocks form a core that is surrounded by an incomplete skin layer of the $F$ block, as illustrated in Fig. 3(b). Since the $O$ blocks protect mainly the $F$ skin, part of the $E$ core is exposed to water. However, when the $O$ block is long enough to curl around to shield the $E$ domain, the core consists of two separate but adjacent domains $(E$ and $F)$, as depicted in Fig. 3(c). When the polymer concentration is high enough, the hamburger micelle $(F-E-F)$ can be formed by merging two $F$ skin layer micelles [Fig. 3(d)]. Its formation can diminish the exposure of the $E$ core of the skin layer micelle to water. Nonetheless, the segmented-worm micelle is never observed. The current experimental approaches might be difficult to identify those micellar morphologies undoubtedly.

Finally, a possible architecture can be obtained by placing the solvophilic $O$ block in the middle of the linear triblock copolymer, that is the linear EOF. For linear EOF (6- $x-7)$ with longer $O$ blocks, a micelle with two neighboring $E$ and $F$ layers is shielded by the $O$ loops, as shown in Fig. 4(b). However, when the $O$ block is too short, only the combination of several two-layer micelles can the solvophobic $E$ 
TABLE I. Summary of the morphologies of micelles observed from the dilute solutions of linear triblock copolymers with various architectures.

\begin{tabular}{cll}
\hline \hline Architecture & \multicolumn{1}{c}{ Morphology } \\
\hline OEF & & Core/shell/corona \\
OFE & Short $O$ & $\begin{array}{l}\text { Incomplete skin-layered micelle } \\
\text { Bicore micelle }\end{array}$ \\
& Long $O$ & Segmented-worm micelle \\
EOF & Short $O$ & Bilayer Micelle \\
& Long $O$ & \\
\hline \hline
\end{tabular}

and $F$ blocks receive enough protection by the $O$ blocks. Consequently, segmented-worm micelles are formed, as shown in Fig. 4(c). Note that there is a difference between the segmented-worm structures formed from the miktoarm star and linear EOF triblock copolymers. The solvophobic $O$ blocks for the segmented-worm micelles of the miktoarm star have free ends, and these ends spread out freely into the solvents. However, the ends of the solvophobic $O$ blocks for the segmented-worm micelles formed by linear EOFs are connected to $E$ and $F$ blocks. Therefore the corona is not as expansive. A summary of the morphologies observed for linear triblock copolymers with different arrangements of the three blocks is presented in Table I.

In summary, copolymers with three chemically distinct blocks are able to form various multicompartment micelles. We have performed dissipative particle dynamics simulations to investigate the effects of molecular architecture, block length, and solution concentration on the morphologies of $A B C$ triblock copolymers. The complex structures within the multidomain micelles are examined. In this work, we have observed the segmented-worm and hamburger micelles for miktoarm star and the core-shell-corona micelle for linear copolymer, which were reported based on experimental observation. ${ }^{3,4,8}$ In addition, we predict the existence of other different morphologies, which can be formed by linear copolymers with different arrangements of the three blocks. Our simulation results indicate that mesoscale dissipative particle dynamics simulation is a powerful tool to explore the morphologies associated with self-assembled aggregates of copolymers and thus can potentially be used to design novel multicompartment micelles.

\section{ACKNOWLEDGMENTS}

This research is supported by National Science Council of Taiwan. Computing time provided by the National Center for High-Performance Computing of Taiwan is gratefully acknowledged.

${ }^{1}$ I. W. Hamley, The Physics of Block Copolymers (Oxford University Press, Oxford, 1998).

${ }^{2}$ A. Laschewsky, Curr. Opin. Colloid Interface Sci. 8, 274 (2003).

${ }^{3}$ Z. Li, E. Kesselman, Y. Talmon, M. Hillmyer, and T. P. Lodge, Science 306, 98 (2004).

${ }^{4}$ Z. Zhou, Z. Li, Y. Ren, M. A. Hillmyer, and T. P. Lodge, J. Am. Chem. Soc. 125, 10182 (2003).

${ }^{5}$ T. P. Lodge, A. Rasdal, Z. Li, and M. A. Hillmyer, J. Am. Chem. Soc. 127, 17608 (2005).

${ }^{6}$ Z. Li, M. A. Hillmyer, and T. P. Lodge, Macromolecules 39, 765 (2006).

${ }^{7}$ M. A. Hillmyer and T. P. Lodge, J. Polym. Sci., Part A: Polym. Chem. 40, 1 (2002)

${ }^{8}$ J.-F. Gohy, N. Willet, S. Varshney, J.-X. Zhang, and R. Jérome, Angew. Chem., Int. Ed. 40, 3214 (2001).

${ }^{9}$ Z. Li, M. A. Hillmyer, and T. P. Lodge, Nano Lett. 6, 1245 (2006).

${ }^{10}$ V. Ortiz, S. O. Nielsen, D. E. Discher, M. L. Klein, R. Lipowsky, and J. Shillcock, J. Phys. Chem. B 109, 17708 (2005).

${ }^{11}$ P. J. Hoogerbrugge and J. M. V. A. Koelman, Europhys. Lett. 19, 155 (1992).

${ }^{12}$ P. Español and P. B. Warren, Europhys. Lett. 30, 191 (1995).

${ }^{13}$ R. D. Groot and P. B. Warren, J. Chem. Phys. 107, 4423 (1997).

${ }^{14}$ MATERIALS STUDIO, a software developed by Accelrys Software Inc.

${ }^{15}$ J. Xia and C. Zhong, Macromol. Rapid Commun. 27, 1110 (2006). 